\title{
Monosodium glutamate is not associated with obesity or a greater prevalence of weight gain over 5 years: findings from the Jiangsu Nutrition Study of Chinese adults
}

\author{
Zumin Shi ${ }^{1,2,3 *}$, Natalie D. Luscombe-Marsh ${ }^{4}$, Gary A. Wittert ${ }^{4}$, Baojun Yuan ${ }^{1}$, Yue Dai ${ }^{1}$, Xiaoqun Pan ${ }^{1}$ \\ and Anne W. Taylor ${ }^{2,3}$ \\ ${ }^{1}$ Department of Nutrition and Foodborne Disease Prevention, Jiangsu Provincial Centre for Disease Control and Prevention, \\ 172 Jiangsu Road, Nanjing 210009, China \\ ${ }^{2}$ Population Research and Outcome Studies Unit, South Australian Department of Health, PO Box 287, 11 Hindmarsh Square, \\ Adelaide, SA 5000, Australia \\ ${ }^{3}$ Discipline of Medicine, University of Adelaide, Frome Road, Adelaide, SA 5000, Australia \\ ${ }^{4}$ Discipline of Medicine and NHMRC Centre of Clinical Research Excellence, University of Adelaide, Frome Road, Adelaide, SA \\ 5000, Australia \\ (Received 15 September 2009 - Revised 9 February 2010 - Accepted 10 February 2010 - First published online 7 April 2010)
}

\begin{abstract}
Animal studies and one large cross-sectional study of 752 healthy Chinese men and women suggest that monosodium glutamate (MSG) may be associated with overweight/obesity, and these findings raise public concern over the use of MSG as a flavour enhancer in many commercial foods. The aim of this analysis was to investigate a possible association between MSG intake and obesity, and determine whether a greater MSG intake is associated with a clinically significant weight gain over 5 years. Data from 1282 Chinese men and women who participated in the Jiangsu Nutrition Study were analysed. In the present study, MSG intake and body weight were quantitatively assessed in 2002 and followed up in 2007. MSG intake was not associated with significant weight gain after adjusting for age, sex, multiple lifestyle factors and energy intake. When total glutamate intake was added to the model, an inverse association between MSG intake and $5 \%$ weight gain was found $(P=0 \cdot 028)$, but when the model was adjusted for either rice intake or food patterns, this association was abolished. These findings indicate that when other food items or dietary patterns are accounted for, no association exists between MSG intake and weight gain.
\end{abstract}

Dietary glutamate: Energy intake: Body weight gain: Human studies: Longitudinal studies

Glutamate, a derivative of the non-essential amino acid, L-glutamate, is the most abundant amino acid found in nature, and it is present, in a free and bound form, in many foods of animal and plant origin. Food additives such as monosodium glutamate (MSG) or hydrolysed vegetable proteins are also significant sources. Glutamate is a major taste component of dietary protein, and its unique taste has been described as 'Umami' ${ }^{(1,2)}$. Evidence from human subjects, rats and nonprimates indicate that free glutamate functions as a signal to regulate protein intake and nutritional status ${ }^{(3-7)}$, as well as other physiological processes, i.e. thermoregulation and energy homeostasis ${ }^{(8)}$.

Great public concern has been expressed in Westernised countries that the widespread use of MSG as a flavour enhancer is not good for health ${ }^{(9)}$. In response to these claims, all clinical and laboratory evidence on the adverse effects of MSG were reviewed by several expert health committees, and their findings concluded that MSG had no long-term serious health consequences ${ }^{(10)}$. Additional concern over the
MSG content of commercial foods has been raised due to reports of a link with overweight/obesity. Studies in monkeys, mice and rats provided some evidence that weight gain may be associated with MSG intake, and this may be independent of an increase in appetite ${ }^{(11-13)}$. For example, weight gain over a 4-month period was greater (compared with controls injected with saline) in male and female mice that were subcutaneously injected immediately post birth with a large dose of $\mathrm{MSG}^{(12)}$. Most probably, the observed weight gain was due to the destruction of several brain regions (including the hypothalamus) that are involved in appetite and energy metabolism. At least among the scientific community, it is accepted that the brain lesions observed in such animal studies resulted from relatively high doses of MSG being injected (i.e. approximately $2 \cdot 5-12 \mathrm{mg} / \mathrm{g}$ ), without food, in the neonatal $\operatorname{period}^{(10,14,15)}$.

Human data obtained from the investigations of the link between MSG and obesity are limited, but the coincident increase in obesity since 1960s in Westernised countries and

Abbreviation: MSG, monosodium glutamate.

* Corresponding author: Dr Zumin Shi, fax +61 88226 6244, email zumins@vip.sina.com 
the addition of MSG to commercially prepared foods continue to fuel public debate. Moreover, a recent cross-sectional study of 752 healthy Chinese reported that the MSG intake was positively associated with BMI, independent of total energy intake or physical activity ${ }^{(16)}$. A major criticism of the present study was that the total free glutamate (specified as glutamic acid) intake of the non-users compared with of the users of MSG was the same, possibly due to the fairly homogenous dietary patterns among the predominantly rural community, or it may have resulted from inaccuracy in the assessment of MSG intake.

Given these limited and discrepant findings, further investigation of a possible association between MSG intake and obesity is warranted. Herein, we report the findings from a large population-based study, the Jiangsu Nutrition Study, where MSG intake and body weight were quantitatively assessed in 2002 and followed up in 2007.

\section{Subjects and methods}

\section{Subjects}

In 2002, China launched a national study in nutrition and health. The data presented in this article are from a subsample of participants from the province of Jiangsu. This subsample represents the current cohort, the Jiangsu Nutrition cohort, where associations between nutrition and other risk factors of non-communicable chronic disease are being investigated $^{(17-19)}$. A rural sample was selected from three randomly selected small towns within each of the six counties of Jiangsu (i.e. Jiangyin, Taichang, Shuining, Jurong, Sihong and Haimen). An urban sample was selected from the capital cities of the two prefectures, Nanjing and Xuzhou; and from each capital city, three streets were randomly selected. The six counties and the two prefectures represented a geographically and economically diverse population. In each town/ street, two villages/neighbourhoods were randomly selected, and ninety households were further selected randomly from each village/neighbourhood. All the members in the households were invited to take part in the study. In addition, one-third of the households were interviewed by a trained health worker about their dietary intake, and all family members aged 3 years and older from these households were invited to have fasting blood samples taken. Written consents were obtained from all the participants. The study was conducted according to the guidelines laid down in the Declaration of Helsinki, and all procedures involving human subjects/patients were approved by Jiangsu Provincial Center for Disease Control and Prevention.

In 2002, height, weight and dietary information were obtained from 2849 adults aged 20 years and above, and fasting plasma glucose was measured. In 2007, an attempt to contact all the original participants was made, but some had either moved to other cities for temporary work or moved to other streets within the urban area. Therefore, of the original 2849 participants, 1682 were identified for follow-up, 1492 $(88.7 \%)$ actually participated in the follow-up interview, and 190 participants refused to participate, and height and weight were obtained from $1282(76.2 \%)$ of them (210 participants finished interview at home, but missed the measurement in the clinic). For the analysis of change in body weight over 5 years, we excluded those participants who had extreme values of weight change (i.e. $>20 \mathrm{~kg}, n 11$ ), and those who had known diabetes, stroke or cancer at baseline ( $n$ 40). Four participants had missing values for MSG intake at baseline. The final sample in the study for weight change consisted of 509 men and 718 women (total $n$ 1227). Compared with the retained participants, those lost to the follow-up were generally younger ( $45.5 v .49 .3$ years), but there were no differences in the mean BMI or energy intake.

\section{Data collection and measurements}

Participants were interviewed at their homes by health workers using a standard questionnaire ${ }^{(18)}$. The health workers were intensively trained in all aspects of data collection.

Dietary intake. In 2002, dietary intake patterns during the previous year were investigated by a series of detailed questions about the usual frequency and quantity of intake of thirty-three food groups and beverages ${ }^{(18,19)}$. The FFQ has been validated ${ }^{(20,21)}$ and reported to be a useful method for the collection of individual food consumption information in face-to-face interviews, but not in self-administered surveys due to the current educational level of the majority of the Chinese population. To determine the amount of MSG and other seasonings consumed by individuals, each household was specifically asked about its usual monthly consumption of these items. Individual consumption of MSG was calculated according to the total amount of MSG consumed in the household divided by the number of individuals per household, and was then adjusted for the proportion of the household energy intake by each individual. Average total glutamate intake was also calculated by adding the glutamate concentrations of all foods/seasonings consumed by an individual per day. Nutrient and vegetable oil intakes were also assessed using a $3 \mathrm{~d}$ weighed food diary which recorded all the foods consumed by each individual on three consecutive days; this was done to confirm the intakes reported from the FFQ data. We did not consider under- and over-reporting of energy intake to be an issue, because upon reviewing the food diaries with the subjects, the health workers would clarify any intake value for a particular food that fell below or above the usual value reportedly consumed by the population within the region. Food consumption data were analysed using the Chinese Food Composition Table ${ }^{(22)}$.

Dietary patterns. Dietary patterns were identified by factor analysis based on food intake measured by the FFQ using standard principal component analysis as described elsewhere $^{(18)}$. In short, four food patterns were obtained - Factor 1 ('macho') was characterised by various kinds of animal foods and alcohol, i.e. foods commonly consumed by men; Factor 2 (the 'traditional' pattern) loaded heavily on rice and fresh vegetables, and inversely on wheat starch; Factor 3 ('sweet tooth') contained cake, milk, yoghurt and drinks; and Factor 4 ('vegetable-rich' pattern) included whole grains, fruits, root vegetables, fresh and pickled vegetables, milk, eggs and fish. The four factors explained $28.5 \%$ of the variance in intake.

Other lifestyle factors. Cigarette smoking was assessed by asking the frequency of daily cigarette smoking. Eating out was assessed by asking whether individuals ate out on a frequent basis, and was coded as yes or no. Alcohol consumption was assessed by asking the frequency and amount of alcohol 
consumed. Questions on daily commuting were grouped into three categories: (1) using motorised transportation, or no work ( 0 min of walking or cycling); (2) walking or bicycling for 1-29 min; (3) walking or bicycling for $\geq 30 \mathrm{~min}$. Daily leisure time physical activity was grouped into three categories: $0,1-29$ and $\geq 30 \mathrm{~min}$. Daily time spent on sedentary activities (viewing television, operating computer, playing video games and reading during leisure time) was classified into four categories: $<1,1-1.9,2-2.9$ and $\geq 3 \mathrm{~h}$. Education was recoded into either 'Low' (illiteracy, primary school), 'Medium' (junior middle school) or 'High' (high middle school or higher) based on six categories of education levels in the questionnaire. Occupation was recoded into 'Manual' or 'Non-manual' based on a question with twelve occupational categories.

Anthropometric measurements. In both 2002 and 2007, anthropometric measurements were obtained by the use of standard protocols and techniques. Body weight was measured in light indoor clothing without shoes to the nearest $100 \mathrm{~g}$. Height was measured without shoes to the nearest $\mathrm{mm}$ using a stadiometer. Waist circumference was measured to the nearest $\mathrm{mm}$ midway between the inferior margin of the last rib and the crest of the ilium, in the mid-auxillary line in a horizontal plane.

\section{Statistics}

MSG intake was recoded into quartiles. Weight change was recoded into three groups: weight loss (loss $\geq 5 \%$ ); weight maintenance (weight change $<5 \%$ ); and weight gain (gain $\geq 5 \%$ ). $\chi^{2}$ Test was used to compare differences between categorical variables, and ANOVA was used to compare differences in continuous variables between the groups. Multilevel mixed-effects linear regression was used to determine the association between MSG intake and weight change adjusted for age, education, occupation, active commuting, leisure time physical activity, smoking, alcohol drinking, eating out and energy intake. Multilevel logistic regression was used to assess the association between MSG intake and the development of a clinically significant weight gain $\geq 5 \%(23,24)$. Household cluster was adjusted in these multivariate models using the xtmelogit command. We tested for linear trend across categories of MSG intake by assigning each participant the median value for the category, and by modelling this value as a continuous variable. Food patterns were also included in the multivariate models to control for the residual confounding, as suggested by Imamura et al. ${ }^{(25)}$. All the analyses were performed using STATA 10 (Stata Corporation, College Station, TX, USA). Statistical significance was considered when $P<0 \cdot 05$ (two sided).

\section{Results}

The mean intake of MSG for the entire population was 3.8 (SD 4.3 ) g/d. Of the 1227 participants, 72 reported no use of MSG, and median intakes across the quartiles were $0 \cdot 8,2 \cdot 0$, 3.7 and $6.9 \mathrm{~g} / \mathrm{d}$, respectively. Table 1 shows the cross-sectional associations between MSG intake and nutrients and specific food items or food groups. MSG intake was positively associated with fat intake $(P<0 \cdot 001)$, but was inversely associated with carbohydrate intake $(P<0 \cdot 001)$. No significant difference in energy and protein intake was found across MSG intake quartiles. Total glutamate was the same in the first, second and third quartiles, but was on average $37 \%$ greater in the fourth quartile $(P<0 \cdot 001)$. Rice intake was greater among individuals in the higher quartiles of MSG intake $(P<0 \cdot 001)$. Intakes of fruit and vegetables were not different across levels of MSG intake. The prevalence of smoking and alcohol drinking was higher among individuals in the higher quartiles of MSG intake $(P<0 \cdot 001)$. Cross-sectionally, there was an inverse association between MSG intake and weight status and BMI; body weight and BMI tended to decrease across quartiles of MSG intake (all $P<0.05$ ). At baseline, the overall prevalence of overweight/obesity $\left(\right.$ BMI $\geq 25 \mathrm{~kg} / \mathrm{m}^{2}$ ) was $29.0 \%$. Only $9.0 \%$ of the sample were obese $\left(\right.$ BMI $\left.\geq 28 \mathrm{~kg} / \mathrm{m}^{2}\right)$. Across quartiles of MSG intake, there was a significant decrease in the prevalence of obesity $(P=0 \cdot 016)$. However, this association existed after adjusting for socio-demographic factors and dietary patterns in logistic regression. OR for obesity across quartiles of MSG were $1,0.91(0.54,1.55), 0.87(0.48,1.58)$ and 0.56 $(0.29,1.09 ; P$ for trend 0.005$)$. When we used all the available data including those lost to follow-up, the baseline association between MSG and obesity was NS: OR across quartiles of MSG intake were $1,0.93(0.66,1.32), 1.01(0.72,1.43)$ and $0.71(0.49,1.04 ; P$ for trend $=0.142$; data not shown $)$.

Table 2 shows the longitudinal associations between MSG intake and changes in body weight status. The mean 5-year weight gain among all the participants was 0.8 (SD 4.7) $\mathrm{kg}$. The prevalence of $5 \%$ weight gain, weight maintenance and weight loss were $26 \cdot 2,59 \cdot 0$ and $14.8 \%$, respectively. No association between MSG intake and status of weight change over 5 years was found. The mean MSG intake was 3.8 (SD 4.0) $\mathrm{g}$ among those having $5 \%$ weight gain. The corresponding figures were 3.7 (SD 5.0) g for $5 \%$ weight loss and 3.9 (SD 4.0) $\mathrm{g}$ for weight maintenance (data not shown). There was, however, a significant difference in the weight and BMI change across quartiles of MSG intake. Compared with those in the first quartile of MSG intake, those in the fourth quartile of MSG intake had lower weight and BMI gain, but had a greater increase in waist circumference. After adjusting for age and sex, there was an inverse association between MSG intake and $5 \%$ weight gain; the OR for $5 \%$ weight gain across quartiles of MSG intake were 1, 0.62 (95\% CI 0.38 , $1.01), 0.48$ (95\% CI $0.29,0.80)$ and 0.58 (95\% CI 0.35 , 0.97; $P$ for trend=0.066). After adjusting for lifestyle, demographic factors, energy intake, sex and total glutamate intake, a linear inverse trend between MSG intake and 5\% weight gain was observed (Model 4, $P=0.028$ ). However, this association disappeared when Model 3 was adjusted for either rice intake $(P=0 \cdot 90)$ or food patterns $(P=0 \cdot 85)$.

In the province, a distinct definition of urban/rural is difficult due to economic development. Since we have adjusted for education and job status plus dietary patterns, we decided not to adjust for urban/rural. Because of the small number of the urban sample, we restricted the sensitivity analysis to the rural sample. The above-described associations did not change when the analyses were limited to the rural sample or to those reporting the main eating place as home. In the multivariate analysis, traditional food pattern was inversely associated with $5 \%$ weight gain (OR $0.50,95 \%$ CI 0.39 , $0.64, P<0 \cdot 001)$. 


\section{Discussion}

To our knowledge, this is the second human study to investigate a possible association between MSG intake and obesity, but more importantly it is the first to determine whether a greater MSG intake is associated with a clinically significant weight gain over 5 years. In contrast to the InterMap study of 752 healthy Chinese men and women ${ }^{(16)}$, we found that the MSG intake in a Chinese population from Jiangsu province was neither associated with obesity nor associated with a clinically significant weight gain (i.e. $\geq 5 \%$ ) after 5 years.

Data from the 2002 National Nutrition and Health Survey showed that $14.7 \%$ of Chinese were overweight (using the WHO definition of BMI $\geq 25 \mathrm{~kg} / \mathrm{m}^{2}$ ), and that another $2.6 \%$ were obese $\left(B M I \geq 30 \mathrm{~kg} / \mathrm{m}^{2}\right)^{(26)}$. In the current Jiangsu province population, the prevalence of overweight and obesity (BMI $\geq 25 \mathrm{~kg} / \mathrm{m}^{2}$ ) was $29.0 \%$ in 2002 , which reflects the relatively high socio-economic status of this region of China. It has been established that China's rapidly increasing prevalence of overweight and obesity is the result of changes in the traditional diet, reduced levels of physical activity and increased sedentary lifestyle. However, in the current Jiangsu sample, the mean weight gain over 5 years was low (only $0.8 \mathrm{~kg}$ ). This finding may have at least two explanations: (i) the socio-economic status of the Jiangsu population is relatively high compared with of other regions of China, and once a high socio-economic status is reached, the prevalence of obesity tends to plateau $^{(26)}$; (ii) the fact that a large proportion of this sample maintained their usual lifestyle over this 5-year period (regardless of whether it was 'traditional' or not), and hence, this indicates that specific populations which can resist the changing environment are less prone to weight gain. It must be acknowledged that the participants who were most likely to have changed their lifestyle were probably

Table 1. Baseline sample characteristics according to monosodium glutamate intake quartiles (Q) among Chinese adults (Mean values with their standard errors)

\begin{tabular}{|c|c|c|c|c|c|c|c|c|c|}
\hline & \multicolumn{2}{|c|}{ Q1 (low, n 307) } & \multicolumn{2}{|c|}{ Q2 (n 307) } & \multicolumn{2}{|c|}{ Q3 (n 307) } & \multicolumn{2}{|c|}{ Q4 (high, $n$ 306) } & \multirow[b]{2}{*}{$P$} \\
\hline & Mean & SE & Mean & SE & Mean & SE & Mean & SE & \\
\hline Energy $(\mathrm{kJ} / \mathrm{d})^{\star}$ & 9706 & 142 & 9898 & 142 & 9643 & 142 & 9765 & 146 & 0.638 \\
\hline Fat $(g / d) \dagger$ & 73.9 & 1.4 & $81 \cdot 3$ & 1.4 & 84.5 & 1.4 & 86.7 & 1.4 & $<0.001$ \\
\hline Protein $(g / d) \dagger$ & $72 \cdot 1$ & 0.8 & $72 \cdot 2$ & 0.8 & $72 \cdot 6$ & 0.8 & $72 \cdot 4$ & 0.8 & 0.974 \\
\hline Carbohydrate $(\mathrm{g} / \mathrm{d}) \dagger$ & 336.5 & $3 \cdot 4$ & $321 \cdot 0$ & $3 \cdot 4$ & $310 \cdot 4$ & $3 \cdot 4$ & $300 \cdot 6$ & 3.4 & $<0.001$ \\
\hline Fibre $(g / d) \dagger$ & $16 \cdot 0$ & 0.5 & 11.8 & 0.5 & $9 \cdot 0$ & 0.5 & 9.5 & 0.5 & $<0.001$ \\
\hline Glutamate $(\mathrm{g} / \mathrm{d}) \dagger$ & $14 \cdot 2$ & 0.3 & $14 \cdot 0$ & 0.3 & $13 \cdot 9$ & 0.3 & $19 \cdot 2$ & 0.3 & $<0.001$ \\
\hline Rice $(g / d)^{*}$ & 238 & 9 & 306 & 9 & 380 & 9 & 357 & 9 & $<0.001$ \\
\hline Wheat starch $(g / d)^{*}$ & 196 & 8 & 106 & 8 & 39 & 8 & 39 & 8 & $<0.001$ \\
\hline Vegetables $(\mathrm{g} / \mathrm{d})^{*}$ & 259 & 9 & 256 & 9 & 259 & 9 & 264 & 9 & 0.946 \\
\hline Fruits $(g / d)^{\star}$ & 57 & 5 & 57 & 5 & 54 & 5 & 59 & 5 & 0.914 \\
\hline Animal foods $(g / d)^{*}$ & 48 & 3 & 58 & 3 & 63 & 3 & 65 & 3 & $<0.001$ \\
\hline Alcohol intake (g/d) & 1.8 & 0.3 & $1 \cdot 7$ & 0.3 & $1 \cdot 7$ & 0.3 & 1.9 & 0.3 & 0.954 \\
\hline Men (\%) & \multicolumn{2}{|c|}{$33 \cdot 2$} & \multicolumn{2}{|c|}{$35 \cdot 2$} & \multicolumn{2}{|c|}{$44 \cdot 0$} & \multicolumn{2}{|c|}{$53 \cdot 6$} & $<0.001$ \\
\hline Age (years) & $48 \cdot 2$ & 0.8 & $50 \cdot 0$ & 0.7 & $49 \cdot 6$ & 0.8 & $48 \cdot 2$ & 0.7 & 0.200 \\
\hline Urban (\%) & \multicolumn{2}{|c|}{$24 \cdot 8$} & \multicolumn{2}{|c|}{16.9} & \multicolumn{2}{|c|}{4.2} & \multicolumn{2}{|c|}{6.5} & $<0.001$ \\
\hline Weight $(\mathrm{kg})^{*}$ & $60 \cdot 3$ & 0.5 & $60 \cdot 4$ & 0.5 & 59.5 & 0.5 & $58 \cdot 5$ & 0.5 & 0.045 \\
\hline Waist circumference $(\mathrm{cm})^{\star}$ & $79 \cdot 8$ & 0.5 & $79 \cdot 3$ & 0.5 & $78 \cdot 4$ & 0.5 & $76 \cdot 9$ & 0.5 & 0.001 \\
\hline Central obesity (\%) $\ddagger$ & \multicolumn{2}{|c|}{$36 \cdot 6$} & \multicolumn{2}{|c|}{32.9} & \multicolumn{2}{|c|}{$27 \cdot 8$} & \multicolumn{2}{|c|}{$21 \cdot 2$} & $<0.001$ \\
\hline Obesity (\%; BMI $\geq 28$ kg/m²) & \multicolumn{2}{|c|}{$12 \cdot 4$} & \multicolumn{2}{|c|}{$10 \cdot 1$} & \multicolumn{2}{|c|}{$8 \cdot 1$} & \multicolumn{2}{|c|}{$5 \cdot 2$} & 0.016 \\
\hline BMI $\left(\mathrm{kg} / \mathrm{m}^{2}\right)^{*}$ & $23 \cdot 6$ & 0.2 & $23 \cdot 6$ & 0.2 & 23.4 & 0.2 & $22 \cdot 9$ & $0 \cdot 2$ & 0.016 \\
\hline \multicolumn{10}{|l|}{ Education (\%) } \\
\hline Low & $58 \cdot 0$ & & $55 \cdot 1$ & & 53.4 & & $46 \cdot 1$ & & \\
\hline Medium & 31.9 & & $36 \cdot 2$ & & $31 \cdot 6$ & & $43 \cdot 5$ & & 0.005 \\
\hline High & $10 \cdot 1$ & & $8 \cdot 8$ & & $15 \cdot 0$ & & $10 \cdot 5$ & & \\
\hline Manual job (\%) & $56 \cdot 4$ & & $53 \cdot 1$ & & $50 \cdot 8$ & & $47 \cdot 4$ & & 0.154 \\
\hline \multicolumn{10}{|l|}{ Active commuting (\%) } \\
\hline None & $37 \cdot 1$ & & $39 \cdot 4$ & & $39 \cdot 1$ & & $46 \cdot 4$ & & \\
\hline $1-29 \mathrm{~min} / \mathrm{d}$ & $46 \cdot 3$ & & $48 \cdot 2$ & & 51.5 & & $48 \cdot 7$ & & $<0.001$ \\
\hline$\geq 30 \mathrm{~min} / \mathrm{d}$ & $16 \cdot 6$ & & $12 \cdot 4$ & & 9.5 & & 4.9 & & \\
\hline Leisure time physical activity & & & & & & & & & \\
\hline None & 89.9 & & $90 \cdot 2$ & & $92 \cdot 2$ & & 94.4 & & \\
\hline $1-29 \mathrm{~min} / \mathrm{d}$ & $4 \cdot 2$ & & $6 \cdot 5$ & & 3.9 & & 2.9 & & 0.130 \\
\hline$\geq 30 \mathrm{~min} / \mathrm{d}$ & $5 \cdot 9$ & & $3 \cdot 3$ & & 3.9 & & $2 \cdot 6$ & & \\
\hline Sedentary activity & & & & & & & & & \\
\hline$<1 \mathrm{~h} / \mathrm{d}$ & $24 \cdot 4$ & & $16 \cdot 3$ & & $10 \cdot 4$ & & $11 \cdot 8$ & & $<0.001$ \\
\hline $1-1.9 \mathrm{~h} / \mathrm{d}$ & $35 \cdot 2$ & & $29 \cdot 6$ & & $30 \cdot 9$ & & $27 \cdot 1$ & & \\
\hline $2-2.9 \mathrm{~h} / \mathrm{d}$ & $31 \cdot 3$ & & $42 \cdot 4$ & & $49 \cdot 2$ & & $44 \cdot 1$ & & \\
\hline$\geq 3 \mathrm{~h} / \mathrm{d}$ & $9 \cdot 1$ & & $11 \cdot 7$ & & $9 \cdot 5$ & & $17 \cdot 0$ & & \\
\hline Smoker (\%) & $23 \cdot 1$ & & $21 \cdot 2$ & & $28 \cdot 7$ & & $36 \cdot 0$ & & $<0.001$ \\
\hline Alcohol drinker (\%) & $19 \cdot 2$ & & $19 \cdot 9$ & & $26 \cdot 1$ & & $34 \cdot 0$ & & $<0.001$ \\
\hline
\end{tabular}

${ }^{*}$ Adjusted for age and sex.

$\dagger$ Adjusted for age, sex and energy intake.

$\ddagger$ Based on International Diabetes Federation definition for Chinese population. 
Table 2. Weight gain (5\%) according to monosodium glutamate (MSG) intake quartiles (Q) among adults in China (Mean values with their standard errors, odds ratios and $95 \%$ confidence intervals)

\begin{tabular}{|c|c|c|c|c|c|c|c|c|c|c|c|c|c|c|c|}
\hline & \multicolumn{2}{|c|}{ Q1 (low) } & \multicolumn{4}{|c|}{ Q2 } & \multicolumn{4}{|c|}{ Q3 } & \multicolumn{4}{|c|}{ Q4 (high) } & \multirow[b]{2}{*}{$P$} \\
\hline & Mean & SE & Mean & SE & OR & $95 \% \mathrm{Cl}$ & Mean & SE & OR & $95 \% \mathrm{Cl}$ & Mean & SE & OR & $95 \% \mathrm{Cl}$ & \\
\hline Number of participants & 307 & & 307 & & & & 307 & & & & 306 & & & & \\
\hline Number of cases with $5 \%$ weight gain & 103 & & 75 & & & & 67 & & & & 78 & & & & \\
\hline Median MSG intake ( $\mathrm{g} / \mathrm{d})$ & 0.8 & & $2 \cdot 0$ & & & & 3.7 & & & & 6.9 & & & & \\
\hline Mean weight change (adjusted for age and sex) & 1.4 & 0.3 & 0.8 & 0.3 & & & -0.01 & 0.3 & & & 1.0 & 0.3 & & & 0.002 \\
\hline Mean BMI change (adjusted for age and sex) & 0.5 & $0 \cdot 1$ & 0.2 & 0.1 & & & 0.04 & $0 \cdot 1$ & & & 0.3 & 0.1 & & & 0.014 \\
\hline $\begin{array}{l}\text { Mean waist circumference change (adjusted for } \\
\text { age and sex) }\end{array}$ & 2.5 & 0.4 & $2 \cdot 0$ & 0.4 & & & 1.8 & 0.4 & & & $3 \cdot 7$ & 0.4 & & & 0.003 \\
\hline Central obesity at follow-up (\%) & 43.5 & & 38.8 & & & & $29 \cdot 0$ & & & & $30 \cdot 4$ & & & & $<0.001$ \\
\hline Multivariate adjusted for age and sex (Model 1$)^{\star}$ & 1 & & & & 0.62 & $0.38,1.01$ & & & 0.48 & $0.29,0.80$ & & & 0.58 & $0.35,0.97$ & 0.066 \\
\hline Model $1+$ lifestyle (Model 2) $†$ & 1 & & & & 0.59 & $0.35,0.97$ & & & 0.46 & $0.27,0.78$ & & & 0.60 & $0.35,1.02$ & 0.110 \\
\hline Model $2+$ energy (Model 3) $\ddagger$ & 1 & & & & 0.59 & $0.36,0.98$ & & & 0.46 & $0.27,0.78$ & & & 0.60 & $0.35,1.03$ & 0.135 \\
\hline Model 3 + glutamate intake (Model 4)§ & 1 & & & & 0.59 & $0.35,0.97$ & & & 0.45 & $0.27,0.78$ & & & 0.47 & $0.27,0.84$ & 0.028 \\
\hline Model 3 + rice intake (Model 5)\| & 1 & & & & 0.73 & $0.44,1.21$ & & & 0.77 & $0.45,1.32$ & & & 0.93 & $0.54,1.60$ & 0.900 \\
\hline Model $3+$ food patterns 1 (Model 6$)^{\star *}$ & 1 & & & & 0.71 & $0.43,1.18$ & & & 0.73 & $0.42,1.26$ & & & 0.94 & $0.54,1.63$ & 0.850 \\
\hline
\end{tabular}

*All the models were adjusted for baseline weight. Household was treated as a cluster variable.

† Multivariate Model 2 was adjusted for variables in Model 1 plus smoking (yes/no), drinking (yes/no), eating out (yes/no), active commuting (no, 1-29 and $\geq 30$ min/d), leisure time physical activity (no, $1-29$ and $>30$ min/d), sedentary activity $(<1,1-9,2-2 \cdot 9$ and $\geq 3 \mathrm{~h} / \mathrm{d})$, education (low, medium and high) and occupation (manual/non-manual).

¥In addition to the factors included in Model 2, Model 3 included an adjustment for energy intake.

§Model 4 included an adjustment for total glutamate intake.

II In addition to the factors included in Model 3, Model 5 included an adjustment for rice intake.

I Derived by factor analysis.
* In addition to the factors included in Model 3 , Model 6 included an adjustment for food patterns. 
those who could not be contacted at follow-up, and this may bias the findings for weight gain over 5 years.

An interesting observation from this population was that when total glutamate intake (i.e. that derived from added MSG plus from other food sources) was incorporated into the change in body weight model (adjusted for age, sex, energy intake and lifestyle), a significant negative association between MSG intake and 5\% weight gain was found. However, when the model was adjusted for dietary patterns, the relationship between total glutamate intake and weight gain was abolished. This highlights the fact that epidemiological observations can be limited because of potential residual confounding by correlated dietary components ${ }^{(25,27)}$. Accordingly, dietary patterns associated with overweight and obesity, and not just individual nutrients, should be considered in multivariate regression models of health outcomes.

Overweight and obesity are usually the result of excess energy intake. Although average energy intake was almost the same in every quartile of MSG intake, the lower quartile had higher weight and waist circumference, than the higher quartile (despite a high intake of fat and low intake of fibre in this quartile). Although there may be other specific factors relating to weight change missed in this population, the inverse association between traditional food pattern and $5 \%$ weight gain shows the importance of overall dietary pattern in weight change.

Just as excess energy intake may cause obesity, being underweight results from deficiencies in energy and protein. A study of underweight, protein and energy malnourished infants found that they preferred higher concentrations of MSG (and also a casein hydrolysate) in soup, and actually consumed a great volume of it, when compared with their respective controls with good nutritional and protein status ${ }^{(4)}$. Although the study did not assess the prolonged effects of MSG-supplemented soup on the body weight gain of infants, its findings implied that such a heightened sensory response to amino acids is an adaptation to relieve a protein deficiency in the first instance. In addition, Essed et al. ${ }^{(28)}$ performed a single-blind, randomised 16-week parallel study in eighty-three apparently well-nourished (mean BMI, $27.8 \mathrm{~kg} / \mathrm{m}^{2}$ ) elderly nursing home patients - they added $700 \mathrm{mg}$ flavour and/or $300 \mathrm{mg}$ MSG to the animal protein part of the cooked lunchtime meal to increase the protein intake of subjects. After 16 weeks, they found that enhancing the taste of the protein component of the meal increased protein intake, but it did not lead to a higher overall energy intake or cause a change in weight (presumably the extra energy consumed as protein was off-set by a reduction in energy obtained from other nutrients, as was shown by Bellisle et $\left.a l{ }^{(29)}\right)$. In the current Jiangsu population, since MSG users in each quartile, and especially those in the top quartile of MSG users, were not, on average, suffering from a deficiency in protein or energy intake, it is of no great surprise that we did not observe an association between the added MSG/total free glutamate concentration and weight change.

Although our findings differ from those of the InterMap study ${ }^{(16)}$, the two populations are not directly comparable for several reasons. The intake of MSG in the current study population (mean of $3.8 \mathrm{~g} / \mathrm{d}$ ) was approximately 11-fold higher than that of MSG in the InterMap population (mean of $0.33 \mathrm{~g} / \mathrm{d}$ ). Discrepant values may be due to an inaccuracy in the measurement of MSG, but it is not possible to determine whether our study overestimated, or the InterMap study underestimated the amount of MSG consumed. Economically, our participants were from a heterogenous, but largely affluent area of China, whereas the InterMap subjects were predominantly from less developed rural areas. The economic development of the study sites in the InterMap study is many years behind that of the rural study sites in the present study. In rural areas with booming economies, consumption of MSG would be expected to be higher than that in poor rural areas due to both affordability and availability. In the InterMap study, MSG consumption was significantly positively associated with animal protein consumption (across levels of MSG consumption, the animal protein intakes (\% energy) were 1.9 , $2 \cdot 3,2.7$ and $3.1 \%, P<0.01$ ), suggesting a positive association between MSG consumption and socio-economic status in rural Chinese populations. Thus, it would be reasonable to see a higher MSG consumption in our sample than in the InterMap sample. Data from Japan, China and Korea indicate that intakes of MSG in these Asian countries are approximately $1.4-1.6 \mathrm{~g} / \mathrm{d}^{(30)}$, and it is acknowledged that highly seasoned restaurant meals can contain as much as $5 \mathrm{~g}$ or more ${ }^{(9)}$. Moreover, consumer data from the United Kingdom indicated a higher mean intake of MSG $(0.6 \mathrm{~g} / \mathrm{d})$ than the InterMap population (with extreme users consuming as much as $2 \cdot 3 \mathrm{~g} / \mathrm{d}$ ) ${ }^{(9)}$. We also considered that discrepant values of MSG intake may be a reflection of varying dietary patterns in the different sampling regions of China; our population was from the south, whereas the InterMap population was mainly from the north (two north areas and one south area). Both rice and wheat are staple foods in South China, whereas wheat starch is a staple food in Northern China. However, wheat starch contains approximately eight times the glutamate concentration of rice (3600 v. $450 \mathrm{mg} / 100 \mathrm{~g}$ dry weight of food), and therefore, it could be expected that the total intake of glutamate for the InterMap population may have been higher than that for our population. Again, this highlights the need to comprehensively adjust regression models for various dietary patterns and not for just selected individual nutrients; to our knowledge, the InterMap results were not adjusted for complex dietary patterns. On the other hand, the InterMap population had a daily energy intake of approximately $8364 \mathrm{~kJ} / \mathrm{d}$, whereas the current sample consumed about $9618 \mathrm{~kJ} / \mathrm{d}$, which accounts for at least a small proportion of the difference.

The main limitation of the study is that the measurement of MSG consumption was done according to the total amount of MSG consumed in the household divided by the number of individuals per household and then adjusted for the proportion of the household energy intake. The method may not be accurate, although in a large epidemiological study, this would be the most practical way of measuring MSG consumption. Other limitations include the inability to account for the change in MSG consumption during the 5-year follow-up period, and the lack of control for medication use, which may affect weight change. Because the overall prevalence of obesity was low in this population, the power to generalise the relationship between MSG intake and obesity was limited. Nevertheless, the large intake variation in MSG and high mean intakes provides the power to study the association between MSG and weight gain. There was a relatively high attrition rate in the study. Loss to follow-up occurred 
mainly because of job migration in rural areas and construction in urban areas. Because the exposure measurements were done at baseline, loss to follow-up is an unlikely source of bias between the exposure variable and outcome variable. However, it does affect the ability to generalise the findings as the baseline association between the MSG intake and obesity differed between those followed up and the general population. More research, especially in different regions and populations, is needed to confirm our findings.

In conclusion, these findings from a Chinese sample from Jiangsu province indicate that an MSG intake that ranges from 2.5 to 9 times greater than the MSG intake of 'nonusers' is not associated with a higher prevalence of obesity, or with a clinically significant weight gain over 5 years, even after adjustment for a number of covariates including dietary patterns. We suggest that further research in human subjects is warranted to determine if dietary glutamate intake is associated with weight gain in individuals who have a poor protein and energy status.

\section{Acknowledgements}

Z. S. had full access to all the data in the study, and takes responsibility for the integrity of the data, the accuracy of the data analysis, reporting of the methodology, interpretation of the data, and critical review of all drafts and submission. N. D. L.-M. is responsible for literature review, data interpretation, the first draft of the paper and all subsequent revisions. B. Y., Y. D. and X. P. are responsible for data collection. All the authors reviewed the manuscript critically. The study is supported by Jiangsu Provincial Natural Science Foundation (BK2008464, principal investigator Z. S.) and the Jiangsu Provincial Health Bureau, China, and by a research fellowship from the National Health and Medical Research Council of Australia, Centre of Clinical Research Excellence in Nutritional Physiology, Interventions and Outcomes (N. D. L.-M.). The authors disclose that they have no relevant financial interest in this article.

\section{References}

1. Kurihara K \& Kashiwayanagi M (2000) Physiological studies on umami taste. J Nutr 130, Suppl. 4, 931S-934S.

2. Yamaguchi S \& Ninomiya K (2000) Umami and food palatability. J Nutr 130, Suppl. 4S, 921S-926S.

3. Smriga M \& Torii K (2000) Release of hypothalamic norepinephrine during MSG intake in rats fed normal and nonprotein diet. Physiol Behav 70, 413-415.

4. Vazquez M, Pearson PB \& Beauchamp GK (1981) Flavour preferences in malnourished Mexican infants. Physiol Behav 28, 513-519.

5. Murphy C (1989) Aging and chemosensory perception of and preference for nutritionally significant stimuli. Ann N Y Acad Sci 561, 251-266.

6. Mori M, Kawada T, Ono T, et al. (1991) Taste preference and protein nutrition and L-amino acid homeostasis in male Sprague-Dawley rats. Physiol Behav 49, 987-995.

7. Laska M \& Hernandez Salazar LT (2004) Gustatory responsiveness to monosodium glutamate and sodium chloride in four species of nonhuman primates. J Exp Zool A Comp Exp Biol 301, 898-905.

8. Kondoh T, Mallick HN \& Torii K (2009) Activation of the gutbrain axis by dietary glutamate and physiologic significance in energy homeostasis. Am J Clin Nutr, (Epublication ahead of print version on July 8).

9. Food Standards Australia New Zealand (2003) Monosodium Glutamate - A Safety Report. Technical Report (Series no. 23), June 2003. http://www.foodstandards.gov.au (accessed 7 July 2009).

10. Walker R \& Lupien JR (2000) The safety evaluation of monosodium glutamate. J Nutr 130, Suppl. 4S, 1049S-1052S.

11. Olney JW \& Sharpe LG (1969) Brain lesions in an infant rhesus monkey treated with monosodium glutamate. Science $\mathbf{1 6 6}$, 386-388.

12. Olney JW (1969) Brain lesions, obesity, and other disturbances in mice treated with monosodium glutamate. Science 164, 719-721.

13. Hermanussen M, Garcia AP, Sunder M, et al. (2006) Obesity, voracity, and short stature: the impact of glutamate on the regulation of appetite. Eur J Clin Nutr 60, 25-31.

14. Monno A, Vezzani A, Bastone A, et al. (1995) Extracellular glutamate levels in the hypothalamus and hippocampus of rats after acute or chronic oral intake of monosodium glutamate. Neurosci Lett 193, 45-48.

15. Kondoh T \& Torii K (2008) MSG intake suppresses weight gain, fat deposition, and plasma leptin levels in male Sprague-Dawley rats. Physiol Behav 95, 135-144.

16. He K, Zhao L, Daviglus ML, et al. (2008) Association of monosodium glutamate intake with overweight in Chinese adults: the INTERMAP Study. Obesity 16, 1875-1880.

17. Shi Z, Hu X, Yuan B, et al. (2006) Association between serum ferritin, hemoglobin, iron intake, and diabetes in adults in Jiangsu, China. Diabetes Care 29, 1878-1883.

18. Shi Z, Hu X, Yuan B, et al. (2008) Strong negative association between intake of tofu and anemia among Chinese adults in Jiangsu, China. J Am Diet Assoc 108, 1146-1153.

19. Shi Z, Hu X, Yuan B, et al. (2008) Vegetable rich food pattern is related to obesity in China. Int J Obes (Lond) 32, 975-984.

20. Zhao W, Hasegawa K \& Chen J (2002) The use of foodfrequency questionnaires for various purposes in China. Public Health Nutr 5, 829-833.

21. Li YP, He YN, Zhai FY, et al. (2006) Comparison of assessment of food intakes by using 3 dietary survey methods. Zhonghua Yu Fang Yi Xue Za Zhi 40, 273-280.

22. Yang Y (2005) Chinese Food Composition Table 2004. Beijing: Peking University Medical Press.

23. Knowler WC, Fowler SE, Hamman RF, et al. (2009) 10-Year follow-up of diabetes incidence and weight loss in the Diabetes Prevention Program Outcomes Study. Lancet 374, 1677-1686, (Epublication ahead of print version).

24. Field AE, Malspeis S \& Willett W (2009) Weight cycling and mortality among middle-aged or older women. Arch Intern Med 169, 881-886.

25. Imamura F, Lichtenstein AH, Dallal GE, et al. (2009) Confounding by dietary patterns of the inverse association between alcohol consumption and type 2 diabetes risk. Am J Epidemiol 170, 37-45.

26. Wu Y (2006) Overweight and obesity in China. BMJ 333, $362-363$.

27. Newby PK \& Tucker KL (2004) Empirically derived eating patterns using factor or cluster analysis: a review. Nutr Rev 62, 77-203.

28. Essed NH, van Staveren WA, Kok FJ, et al. (2007) No effect of 16 weeks flavour enhancement on dietary intake and nutritional status of nursing home elderly. Appetite 48, 29-36.

29. Bellisle F, Dalix AM, Chapppuis AS, et al. (1996) Monosodium glutamate affects mealtime food selection in diabetic patients. Appetite 26, 267-275.

30. Loliger $\mathbf{J}$ (2000) The use and utility of glutamate as flavouring agents in foods: function and importance of glutamate for savoury foods. J Nutr 130, 915S-920S. 